\title{
Tracing the uptake of gold in Toxoplasma gondii using STEM-EDS
}

Li Xing ${ }^{1}$, Jian-Guo Zheng ${ }^{1}$, Toshihiro Aoki ${ }^{1}$ and Rosa Andrade ${ }^{2}$,

1. Irvine Materials Research Institute, University of California at Irvine, Irvine, CA

2. School of Medicine, University of California at Irvine, Irvine, CA

Approximately 30-50\% of global population is infected with Toxoplasma gondii [1] and current treatments are more than 50 years old. New more efficacious and less toxic treatments are needed. We have previously demonstrated that auranofin, a gold containing drug, has anti-T. gondii activity [2]. While auranofin anti-T.gondii mechanism of action is still unknown, there is enough evidence suggesting that auranofin's gold molecule is responsible for its anti-parasitic effects. Specific drug delivery is fundamental to ensure treatment efficacy and reduce considerable toxicity in host cells, therefore, elucidating whether gold enters the target parasite is paramount. Transmission electron microscopy, because of its powerful nanometer lateral resolution, is an advantageous tool in analyzing the distribution of drugs inside a cell or an infectious pathogen. Herein, we used scanning transmission electron microscopy-energy dispersive Xray spectroscopy (STEM-EDS) to study the uptake of gold(I) by $T$. gondii extracellular tachyzoites treated with auranofin. Different to TEM, scanning transmission electron microscopy (STEM) uses convergent beam scanning over the specimen and an X-ray spectrometer detects the characteristic X-rays emitted by atoms in a sample. Energy-dispersive X-ray spectroscopy (EDS) is therefore a suitable technique to chemically characterize the uptake of gold inside T.gondii and to demonstrate gold entry into the parasite.

To demonstrate the feasibility of STEM-EDS in tracing gold uptake in $T$. gondii tachyzoites, we first examined the distribution of gold after incubation of extracellular T.gondii tachyzoites with auranofin for $2 \mathrm{hrs}$. We found crescent-shape T.gondii extracellular tachyzoites measuring 5um x 2um by STEM. Using uranyl acetate negative staining, we observed the distinctive rhoptry network in the apical end of the parasite in both dark field and bright field image (Fig 1). In the EDS spectrum, a signal peak was detected corresponding to gold inside a T.gondii tachyzoite after two hours incubation with auranofin (solvent control: ethanol), while no peak was observed in its respective control (Fig 2). The peaks corresponding to copper and uranium added during EM grid preparation, were well identified from the spectrum.

This result suggests that STEM-EDS is able to detect the uptake of gold(I) by T.gondii tachyzoites. Our future experiments will determine the location of gold and dynamics of gold uptake in intracellular parasites.

\section{References:}

[1] J Flegr et al, PLoS ONE. 9 (2014), e90203.

[2] R Andrade et al, PLoS NTD 8 (2014), e2973 

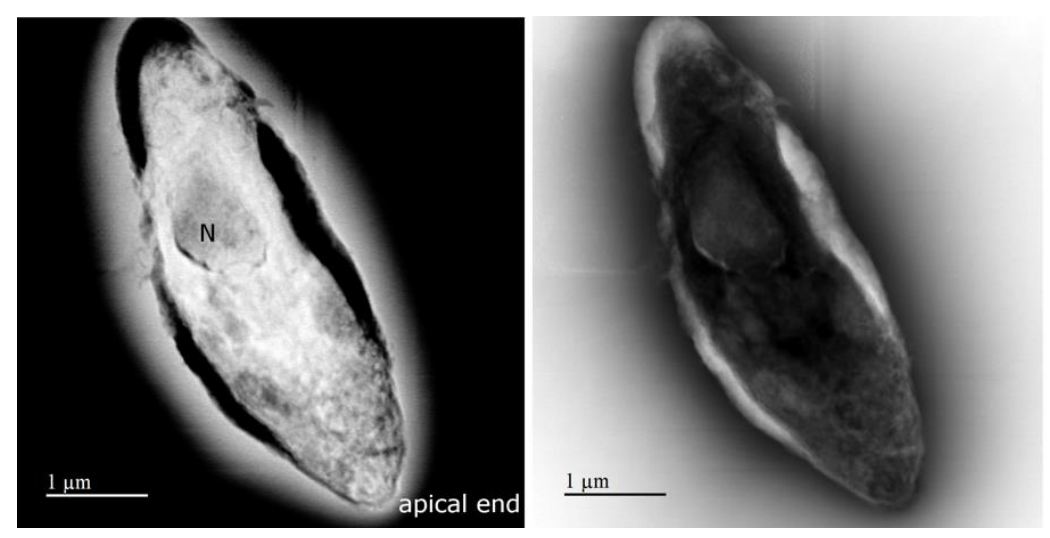

Figure 1. STEM images of Toxoplasma gondii showing internal structures: nucleus (N) and the rhoptry network around apical end. The structure is labelled on the dark field image (left panel).
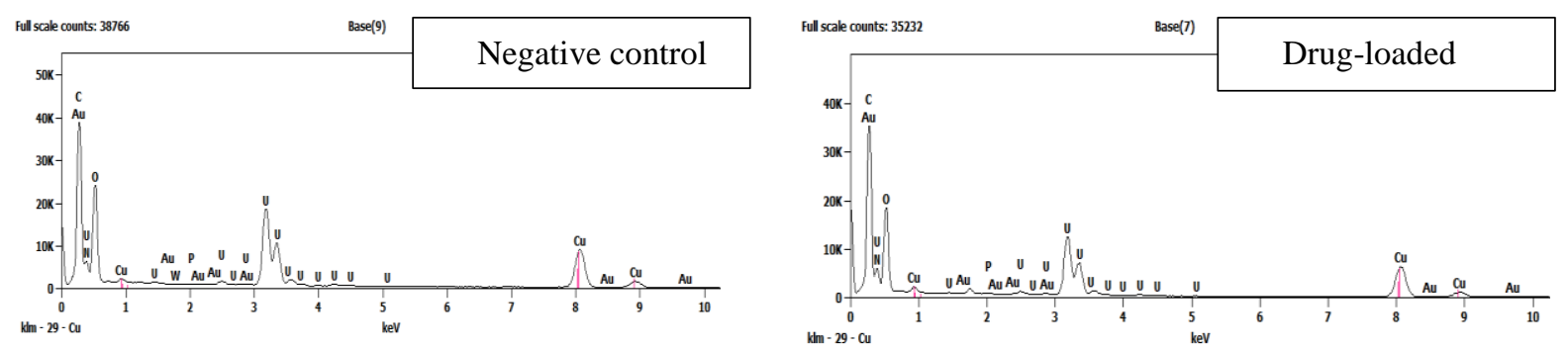

Figure 2. EDS spectra of Toxoplasma gondii tachyzoite incubated with ethanol (negative control) and with auranofin (drug group) 